OPEN ACCESS

Edited by:

Weien Yuan,

Shanghai Jiao Tong University, China

Reviewed by:

Hideki Kitaura,

Tohoku University, Japan

Nathan Pavlos,

University of Western Australia,

Australia

*Correspondence:

Eiichi Hinol

hinoi@p.kanazawa-u.ac.jp

tThese authors have contributed equally to this work.

Specialty section: This article was submitted to Integrative and Regenerative Pharmacology, a section of the journal

Frontiers in Pharmacology

Received: 29 December 2018 Accepted: 27 May 2019

Published: 13 June 2019

Citation:

Hiraiwa M, Ozaki K, Yamada T, lezaki T, Park G, Fukasawa K, Horie T, Kamada H, Tokumura K, Motono M, Kaneda K and Hinoi E (2019) mTORC1 Activation in Osteoclasts

Prevents Bone Loss in a Mouse Model of Osteoporosis.

Front. Pharmacol. 10:684. doi: 10.3389/fphar.2019.00684

\section{mTORC1 Activation in Osteoclasts Prevents Bone Loss in a Mouse Model of Osteoporosis}

\author{
Manami Hiraiwa ${ }^{1 \dagger}$, Kakeru Ozaki ${ }^{1 \dagger}$, Takanori Yamada ${ }^{1}$, Takashi lezaki ${ }^{1,2}$, Gyujin Park' ${ }^{1}$, \\ Kazuya Fukasawa', Tetsuhiro Horie', Hikari Kamada', Kazuya Tokumura1, Mei Motono', \\ Katsuyuki Kaneda ${ }^{1}$ and Eiichi Hinoi ${ }^{1 *}$ \\ ${ }^{1}$ Laboratory of Molecular Pharmacology, Division of Pharmaceutical Sciences, Kanazawa University Graduate School, \\ Kanazawa, Japan, ${ }^{2}$ Venture Business Laboratory, Organization of Frontier Science and Innovation, Kanazawa University, \\ Kanazawa, Japan
}

The mechanistic/mammalian target of rapamycin (mTOR) is widely implicated in the pathogenesis of various diseases, including cancer, obesity, and cardiovascular disease. Bone homeostasis is maintained by the actions of bone-resorbing osteoclasts and bone-forming osteoblasts. An imbalance in the sophisticated regulation of osteoclasts and osteoblasts leads to the pathogenesis as well as etiology of certain metabolic bone diseases, including osteoporosis and osteopetrosis. Here, we identified mTOR complex 1 (mTORC1) as a pivotal mediator in the regulation of bone resorption and bone homeostasis under pathological conditions through its expression in osteoclasts. The activity of mTORC1, which was indicated by the phosphorylation level of its downstream target p70S6 kinase, was reduced during osteoclast differentiation, in accordance with the upregulation of Hamartin (encoded by tuberous sclerosis complex 1 [TSC1]), a negative regulator of $m T O R C 1$. Receptor activator of nuclear factor-kB ligand (RANKL)-dependent osteoclastogenesis was impaired in Tsc1-deficient bone marrow macrophages. By contrast, osteoclastogenesis was markedly enhanced by Raptor deficiency but was unaffected by Rictor deficiency. The deletion of TSC1 in osteoclast lineage cells in mice prevented bone resorption and bone loss in a RANKL-induced mouse model of osteoporosis, although neither bone volume nor osteoclastic parameter was markedly altered in these knockout mice under physiological conditions. Therefore, these findings suggest that mTORC1 is a key potential target for the treatment of bone diseases.

Keywords: metabolic bone diseases, mTORC1, osteoclastogenesis, Raptor, tuberous sclerosis complex 1

\section{INTRODUCTION}

The mechanistic/mammalian target of rapamycin (mTOR) is a kinase belonging to the phosphoinositide 3-kinase-related kinase (PIKK) family of protein kinase. mTOR is composed of two forms of distinct complexes designated as mTOR complex 1 (mTORC1) and mTORC2 (Brown et al., 1994; Sabatini et al., 1994). It is widely implicated in various cellular functions such as growth, proliferation, survival, autophagy, differentiation, and cytoskeletal organization (Sabatini, 2017). The Raptor subunit belongs to mTORC1. Tuberous sclerosis complex 1 (Tsc1) and complex 2 (Tsc2), which encode Hamartin and Tuberin, respectively, are critical negative regulators of mTORC1 through their GTPase-activating 
protein activity toward the small G-protein Ras homolog enriched in the brain (Nojima et al., 2003; Long et al., 2005). On the contrary, the Rictor subunit belongs to mTORC2 (Jacinto et al., 2004). Although global deletion of mTOR, Raptor, and Rictor results in embryonic lethality, cell-specific deletion strategies show that mTOR is implicated in the pathogenesis of various diseases, including cancer, obesity, and cardiovascular disease (Murakami et al., 2004; Guertin et al., 2006; Shiota et al., 2006).

Mesenchymal cells differentiate into skeletal elements by forming a cartilaginous model, which induces bone formation through endochondral ossification in the vertebral column and long bones (Karsenty et al., 2009). Endochondral ossification is required for proper skeletal development and bone modeling, while skeleton integrity, as well as bone remodeling, is believed to be coordinately regulated by two different types of cells, boneforming osteoblasts and bone-resorbing osteoclasts (Harada and Rodan, 2003; Teitelbaum and Ross, 2003). An imbalance in the sophisticated regulation of osteoclasts and osteoblasts leads to pathogenesis as well as the etiology of certain metabolic bone diseases such as osteoporosis, osteopetrosis, and rheumatoid arthritis (Feng and McDonald, 2011).

Studies on genetic mouse have revealed a critical role of mTORC1 in skeletal development through its expression in mesenchymal stem cells or chondrocytes (Chen and Long, 2014; Yan et al., 2016). Additionally, our recent study showed the critical role of mTORC1 in skeletogenesis through the translational control of Sox9 RNA in mesenchymal stem cells (Iezaki et al., 2018). In addition to its role in skeletal development, mTORC1 is essential for the maintaining bone homeostasis through its expression in bone-forming osteoblasts and boneresorbing osteoclasts (Chen and Long, 2018). Although several independent lines of evidence based on pharmacological and genetic strategies show that mTOR signal is important for osteoclast differentiation and function in vitro and in vivo, the precise role of mTOR in osteoclastogenesis is controversial and unknown (Chen et al., 2015; Dai et al., 2017; Zhang et al., 2017; Chen and Long, 2018; Huynh and Wan, 2018).

\section{MATERIALS AND METHODS}

\section{Materials}

Glutathione S-transferase (GST)-receptor activator of nuclear factor- $\kappa \mathrm{B}$ ligand (RANKL) vector and PLAT-E cells were obtained from Dr. S.L. Teitelbaum (Washington University, St. Louis, MO, USA) and T. Kitamura (Tokyo University, Tokyo, Japan), respectively. pMSCVpuro-Cre (\#34564) was obtained from Addgene (Watertown, MA, USA). Recombinant mouse RANKL and macrophage colony-stimulating factor (M-CSF) were purchased from R\&D Systems (Minneapolis, MN, USA). C-terminal Peptide of Type I Collagen (CTx) Enzyme-linked immunosorbent assay (ELISA) kit was obtained from Immunodiagnostic Systems (Boldon, UK). Antibodies were from the following companies: anti- $\beta$-actin was from Santa Cruz Biotechnology (Santa Cruz, CA, USA); anti-mTOR, anti-Raptor, anti-Rictor, anti-Hamartin, anti-pp70S6K1, and anti-p70S6K1 were from Cell Signaling Technology (Danvers, MA, USA). THUNDERBIRD SYBR quantitative polymerase chain reaction (qPCR) Mix was supplied by TOYOBO (Osaka, Japan). Other chemicals used were all of the highest purity commercially available.

\section{Mice}

The protocol used here meets the guideline of the Japanese Society for Pharmacology and was approved by the Committee for Ethical Use of Experimental Animals at Kanazawa University. Raptor $^{f l / f l}$, Rictor ${ }^{f l / f l}$, and Tsc1 ${ }^{f l f l}$ mice were obtained from Jackson laboratory. Tsclflfll mice were crossed with Lyz2-Cre mice to generate $\mathrm{Lyz2}$-Cre; $T s c 1^{f l++}$ mice, and the resulting progenies were intercrossed to obtain $\mathrm{Lyz2}-\mathrm{Cre}$; Tsc $1^{f l f l}$ mice. These mutant mice were backcrossed more than five generations with C57BL/6J. Mice were bred under standard animal housing conditions at $23 \pm 1{ }^{\circ} \mathrm{C}$ with humidity of $55 \%$ and a light/dark cycle of $12 \mathrm{~h}$, with free access to food and water. Genotyping was performed by PCR using tail genomic DNA. The numbers of animals used per experiment are stated in the figure legends.

For generation of an osteoclast-activated osteoporosis model mouse, 8-week-old mice were intraperitoneally administrated GST-RANKL fusion protein daily for 2 days. Mice were killed by decapitation under deep anesthesia with chloral hydrate (400 mg/kg, intraperitoneal injection) $12 \mathrm{~h}$ after final injection (Tomimori et al., 2009; Iezaki et al., 2016).

\section{Bone Histomorphometric Analyses}

Bone histomorphometric analyses were performed on vertebrae not decalcified as previously described (Yamamoto et al., 2012). Briefly, vertebrae were fixed with $10 \%$ formalin, followed by dehydration in different concentrations of ethanol and subsequent embedding in methyl methacrylate resin according to standard protocols. The bone volume to tissue volume ratio $(\mathrm{BV} / \mathrm{TV})$ ratio was measured by von Kossa staining. The bone formation rate (BFR) was analyzed by the calcein double-labeling method. Calcein was injected to mice twice with an interval of 3 days, and then mice were killed 2 days after the last injection. Osteoblast and osteoclast parameters were analyzed by staining with toluidine blue and with tartrate-resistant acid phosphatase (TRAP), respectively. Analyses were performed using the OsteoMeasure Analysis System (OsteoMetrics) according to standard protocols (Hinoi et al., 2007).

\section{Retroviral Transfection}

Retroviral vectors were transfected into PLAT-E cells using the calcium carbonate method. Virus supernatants were collected $48 \mathrm{~h}$ after transfection, and then cells were infected with virus supernatants for $72 \mathrm{~h}$ in the presence of $4 \mu \mathrm{g} / \mathrm{mL}$ of polybrene. Cells were then subjected to selection by culture with $1 \mu \mathrm{g} / \mathrm{mL}$ of puromycin for 3 days before usage for experiments (Fukasawa et al., 2016).

\section{Culture of Osteoclasts and TRAP Staining, the Pit Formation Assay, and the Actin Ring Formation Assay}

Bone marrow macrophages (BMMs) were seeded on 48-well plates (Nunc) at a density of $2.5 \times 10^{4}$ cells/well and were cultured in the 
presence of $20 \mathrm{ng} / \mathrm{mL}$ of M-CSF (100 ng/mL of M-CSF for BMMs infected with retrovirus) and $20 \mathrm{ng} / \mathrm{mL}$ of RANKL for 4 days consecutively (Wang et al., 2006). TRAP staining, the pit formation assay, and the actin ring formation assay were performed as previously described (Iezaki et al., 2016). Briefly, cells were cultured on bone slices and subsequent fixation with $4 \%$ paraformaldehyde, followed by permeabilization with $0.5 \%$ Triton X-100. For the pit assay, the bone slices were scrubbed with a brush to remove attached cells. The bone slices were then treated with $20 \mu \mathrm{g} / \mathrm{mL}$ of lectin solution and subsequent incubation with $0.5 \mathrm{mg} / \mathrm{mL}$ of 3,3'-diaminobenzidine (DAB), including $0.01 \% \mathrm{H}_{2} \mathrm{O}_{2}$.

\section{Isolation of Osteoclasts}

Isolation of osteoclasts was performed from long bones as previously described (Fukasawa et al., 2016). In brief, the long bones were minced into small pieces, and cells were dissociated from bone fragments by vigorous vortex in Alpha modification of Eagle's MEM (aMEM). After removal of bone fragments by sedimentation under normal gravity, the unfractionated bone cells were seeded on collagen gel and were incubated in aMEM with 5\% Fetal bovine serum (FBS). The cells were sequentially treated with $0.001 \%$ Pronase E and $0.02 \%$ EDTA, $0.01 \%$ collagenase, and $0.1 \%$ collagenase. Cells released by final treatment were collected and cultured as osteoclasts.

\section{Real-Time-Based Quantitative PCR}

Total RNA was extracted from cells, followed by synthesis of cDNA with reverse transcriptase and oligo-dT primer. The cDNA samples were then used as templates for real-time PCR analysis, which was performed on an Mx3005P instrument (Agilent Technologies), by using specific primers for each gene (Table 1). Expression levels of the genes examined were normalized by using the $36 \mathrm{~b} 4$ expression levels as an internal control for each sample (Nakamura et al., 2013).

\section{Immunoblotting Analysis}

Cultured cells were solubilized in lysis buffer containing $1 \%$ Nonidet P-40. Samples were then subjected to sodium dodecyl sulfate-polyacrylamide gel electrophoresis (SDS-PAGE), followed by transfer to polyvinylidene difluoride (PVDF) membranes and subsequent immunoblotting assay. Quantification was performed by densitometry using ImageJ.

\section{Data Analysis}

All results are expressed as the mean \pm standard error of the mean, and statistical significance was determined using the

TABLE 1 | List of primers used for real-time PCR.

\begin{tabular}{|c|c|c|}
\hline Genes & Upstream $\left(5^{\prime}-3^{\prime}\right)$ & Downstream $\left(5^{\prime}-3^{\prime}\right)$ \\
\hline Acp5 & CACTCCCACCCTGAGATTTGT & CATCGTCTGCACGGTTCTG \\
\hline Calcr & GTCACTCCTTGTCGATTGCTG & GTTCCCACTGCATTGTCCACA \\
\hline Ctsk & GAAGAAGACTCACCAGAAGCAG & TCCAGGTTATGGGCAGAGATT \\
\hline $36 \mathrm{~b} 4$ & GAGGAATCAGATGAGGATATGGGA & AAGCAGGCTGACTTGGTTGC \\
\hline
\end{tabular}

two-tailed Student $t$-test, and the one-way or two-way ANOVA with Bonferroni/Dunnett post hoc test.

\section{RESULTS}

\section{Expression Level of mTORC1-Related Factors in Primary Cultured Osteoclasts}

To evaluate the validity of primary cultured osteoclasts, TRAP staining and qPCR analysis were performed. TRAP-positive cells were increased in proportion to the culture period up to 4 days (Figure 1A). qPCR analysis revealed that the expression of all osteoclastic marker genes, including cathepsin $\mathrm{K}$ (Ctsk), calcitonin receptor (Calcr), and acid phosphatase 5 (Acp5), was drastically increased during culture from 0 to 4 days (Figure 1B-D). We next examined protein level of mTORC1-related factors in cultured osteoclasts (Figure 1E). The expression of mTOR and Raptor in osteoclasts was stable throughout the differentiation period (Figure 1F and G), whereas the expression of Hamartin (Tsc1) was markedly increased in osteoclasts 2 days after RANKL stimulation, and the increase was sustained thereafter (Figure 1H). In accordance with the upregulation of Hamartin ( $T s c 1$ ) expression, the phosphorylation level of p70S6 kinase (p70S6K), a downstream effector of mTORC1 signaling, was markedly abolished in osteoclasts 2 days after RANKL stimulation (Figure 1I), indicating a possible inverse correlation between Hamartin (Tsc1) expression and p70S6K phosphorylation during osteoclastogenesis. These results suggest that mTORC1 activity in vitro is reduced during osteoclast differentiation in a Hamartin (Tsc1)-dependent manner.

\section{Osteoclast Differentiation is Regulated by mTORC1 but not by mTORC2 In Vitro}

We then evaluated whether the regulators of mTOR pathway control osteoclastogenesis through expression in osteoclasts in vitro. Tsc1 ${ }^{f l / f l}$, Raptor ${ }^{f l / f l}$, and Rictor ${ }^{f / f l}$ mice-derived BMMs were infected with a retrovirus expressing Cre recombinase and subjected to differentiation by RANKL, followed by TRAP staining and pit formation assay. The expression of Hamartin (Tsc1), Raptor, and Rictor was markedly repressed in retrovirusinfected BMMs (Figure 2A-C). The number of TRAP-positive multinucleated cells and area of pit formation were significantly decreased in Tsc1-deleted BMMs but significantly increased in Raptor-deleted BMMs compared with those in control cells (Figure 2D, E, G, H, I, K). By contrast, the number of TRAPpositive multinucleated cells and area of pit formation were comparable between control cells and Rictor-deleted BMMs (Figure 2F, G, J, K). Moreover, the number of actin rings formed was significantly decreased in Tsc1-deleted BMMs (Supplemental Figure 1A and 1B), indicating that mTORC1, rather than mTORC2, controls osteoclast differentiation in a cell-autonomous manner. Conversely, Tsc1 deficiency did not alter the area of pit formation when the same number of mature osteoclasts was used for pit formation assay (Figure $2 \mathbf{L}$ and $\mathbf{M}$ ), suggesting that $T s c 1$ regulates osteoclast differentiation rather than maturation in vitro. 


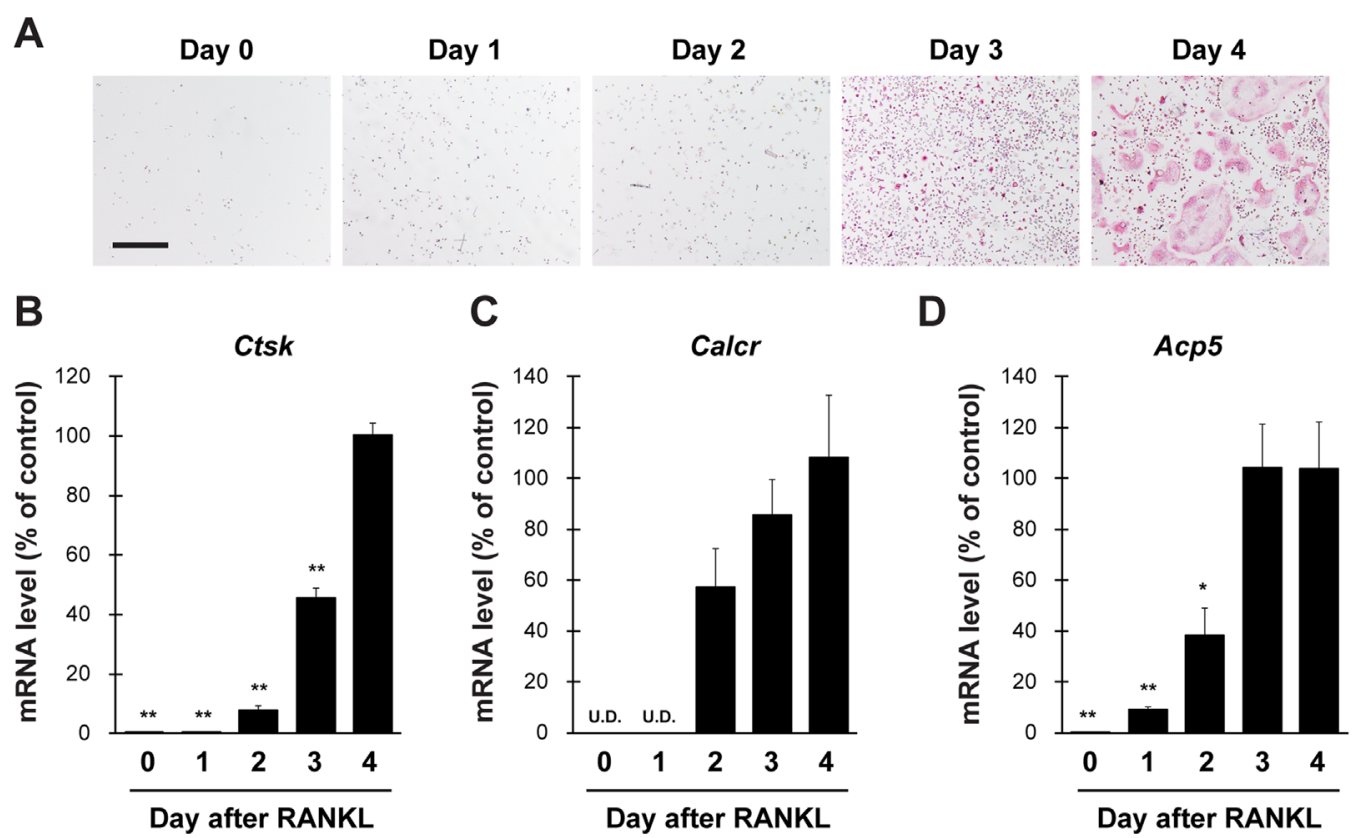

E

Day after RANKL
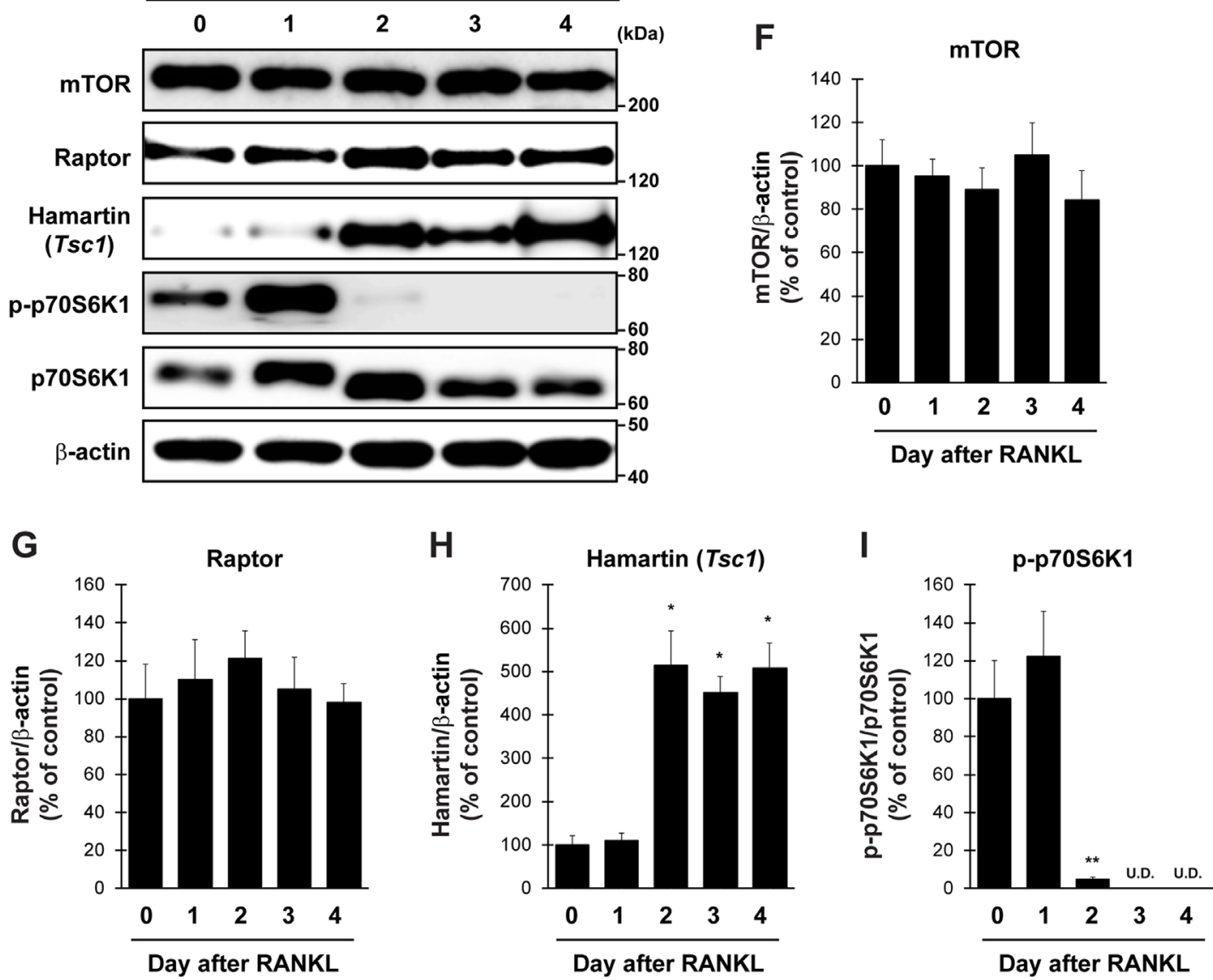

FIGURE 1 | Expression profile of mTORC1-related factors in primary cultured osteoclasts. BMMs prepared from naive mice were stimulated with RANKL and M-CSF, followed by (A) TRAP staining and (B-D) determination of mRNA expression of osteoclastic marker genes by qPCR at day 0 to day 4 ( $n=4)$. (E-I) BMMs from naive mice were stimulated with RANKL, followed by determination of protein expression of mTORC1-related factors by immunoblotting at day 0 to day 4 ( $n=3$ ). All data were analyzed by the one-way ANOVA with Bonferroni/Dunnett post hoc test. ${ }^{\star} P<0.05,{ }^{\star \star} P<0.01$, significantly different from the value obtained in cells at (B-D) day 4 or (F-I) day O. U.D., under detection. Bar $=100 \mu \mathrm{m}$. 


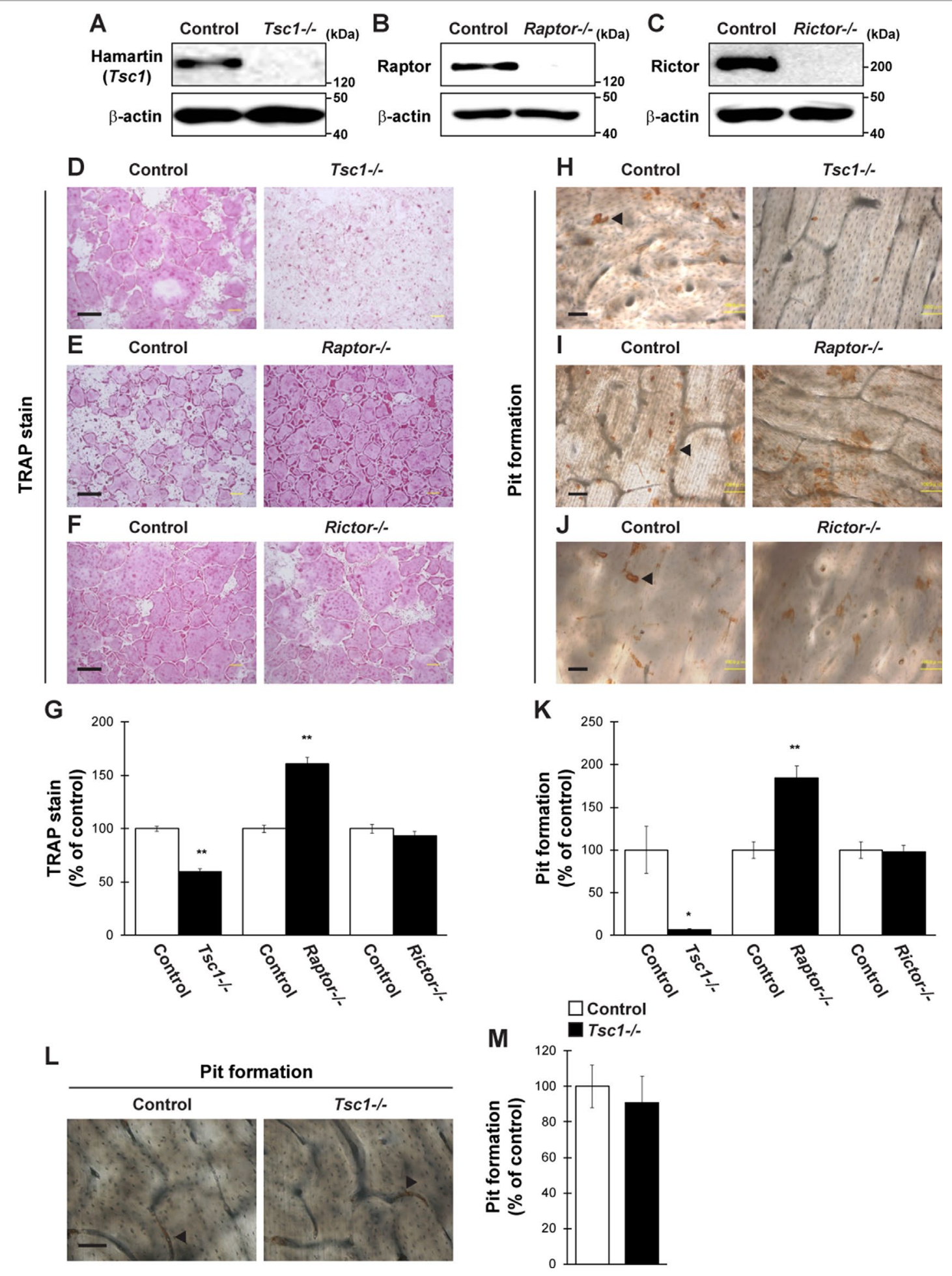

FIGURE 2 | Osteoclastogenesis is regulated by mTORC1 activity. (A-C) BMMs from Tsc1f/ffl mice, Raptorfl/fl mice, or Rictorfl/fl mice were retrovirally infected with Cre recombinase, followed by determination of Hamartin (Tsc1), Raptor, or Rictor expression ( $n=3)$. (D-K) BMMs from Tsc1fl/fl mice, Raptorfl/fl mice, or Rictorfl/fl mice were retrovirally infected with Cre recombinase and subsequent stimulation with RANKL, followed by performing (D-G) TRAP staining and (H-K) pit formation assay $(n=3)$. (L and $\mathbf{M})$ Osteoclasts prepared from Tsc1flffl mice were retrovirally infected with Cre recombinase and subsequent pit formation assay $(n=3)$. $(\mathbf{H}-\mathbf{J}$ and $\mathbf{L})$ Black arrowheads indicate the representative resorption pit, and the brown staining is due to the DAB staining used in the procedure. All data were analyzed by the two-tailed Student $t$-test. ${ }^{*} P<0.05,{ }^{* *} P<0.01$, significantly different from the value obtained in cells infected with empty vector. Bar $=100 \mu \mathrm{m}$. 


\section{Conditional Deletion of Tsc1 in Osteoclasts Does Not Affect Bone Phenotype Under Normal Physiological Conditions}

To evaluate the physiological importance of Tsc1 in bone homeostasis in vivo, we generated osteoclast lineage cell-specific $T s c 1$ knockout mice by crossing Tsc $1^{f l / f l}$ mice with Lyz2-Cre mice. The physical appearance, body weight, and naso-anal length of mice lacking Tsc1 in Lyz2-expressing cells (hereafter referred to as $L y z 2-C r e ; T s c 1^{f l f l}$ mice) were normal than in control mice (data not shown). In the BMMs of Lyz2-Cre; Tsc $1^{f l f l}$ mice, Hamartin (Tsc1) expression was significantly abolished, and the level of phosphorylated p70S6K was significantly increased, indicating an increase in mTORC1 activity in osteoclast lineage cells (Figure $3 \mathrm{~A}$ and $\mathbf{B}$ ). The BV/TV of $L y z 2-C r e ; T s c 1^{f l / f l}$ mice was indistinguishable from that of control mice in vertebrae at any age examined (Figure 3C and D). Bone histomorphometric analyses revealed that indices of osteoclastic function including osteoclast surface (Oc.S/BS) and osteoclast number (N.Oc/B.Pm) (Figure 3E-G), in addition to bone formation indices such as BFR and osteoblast number (N.Ob/T.Ar), were comparable between Lyz2$\mathrm{Cre} ; \mathrm{Tsc}^{\mathrm{fllfl}}{ }^{\mathrm{mice}}$ and control mice (Figure $\mathbf{3 H}-\mathbf{J}$ ). These results show that Tsc1 deletion in osteoclast lineage cells does not affect bone volume or indices of osteoblastic and osteoclastic function under physiological conditions.

\section{Conditional Deletion of Tsc1 in Osteoclasts Prevents RANKL-Induced Osteoclast Activation and Bone Loss}

Osteoclast lineage-specific knockout of Tsc1 did not cause any abnormalities in bone homeostasis under physiological conditions (Figure 3). However, osteoclast differentiation was markedly impaired in Tsc1-deficient BMMs (Figure 2). This suggests that mTORC1 activation in osteoclasts affects osteoclastogenesis and bone homeostasis under pathological conditions. To test this hypothesis, we investigated the possibility of the involvement of Hamartin (Tsc1) expressed by osteoclasts in the pathogenesis of osteoporosis mouse model using GSTRANKL fusion protein injections. RANKL was injected in control and $L y z 2-C r e ; T s c 1^{f / f l}$ mice daily rate over 2 days, followed by bone phenotype analysis. RANKL injection induced marked bone loss in the vertebrae of control mice but not in those of Lyz2-Cre; Tsc $1^{f l f l}$ mice, and RANKL-induced bone loss was markedly impaired in Lyz2-Cre; Tsc $1^{f l f l}$ mice (Figure 4A and B). RANKL-induced increase in osteoclastic parameter (Oc.S/BS) was observed in control mice but not in Lyz2-Cre; Tsc $1^{f l f l}$ mice (Figure 4C and D). In addition, RANKL treatment increased the serum TRAP and CTx levels in control mice but not in Lyz2Cre; $T s c 1^{f l / f l}$ mice (Figure $4 \mathrm{E}$ and F), suggesting that Hamartin (Tsc1) could be implicated in the osteoclastogenesis and bone resorption in vivo under pathological conditions.

\section{DISCUSSION}

Genetic studies have recently revealed the role of mTORC1 in the cellular function of osteoclasts in vitro and bone homeostasis in vivo; however, the results of these studies have been inconsistent. Previously, inactivation of mTORC1 signaling in osteoclasts has been shown to increase bone mass in mice by inhibiting osteoclast differentiation due to the deletion of Raptor by CtskCre, whereas a decrease in bone mass has been observed by augmenting osteoclast differentiation in mice by deleting Raptor with Lyz2-Cre (Dai et al., 2017; Zhang et al., 2017). Conversely, Tsc1 deletion by Lyz2-Cre increases bone mass and reduces bone resorption (Zhang et al., 2017; Huynh and Wan, 2018). In addition, conditional deletion of $T s c 1$ in hematopoietic stem cells using Vav1-Cre results in high bone mass as a consequence of the impairment of osteoclastogenesis (Huynh and Wan, 2018). Contrary to previous findings, we did not observe any bone phenotypes in Lyz2-Cre; $T s c 1^{f l / f l}$ mice under physiological conditions at any age examined, irrespective of the deletion efficiency (Figure 3). The use of different genetic background and housing condition may also contribute to discrepancies between our results and those of previous studies. Nonetheless, our study shows that the serine/threonine kinase MTORC1 regulates bone resorption and bone remodeling under pathological conditions with activated osteoclasts, through its expression in osteoclast lineage cells.

In this study, we showed that osteoclast differentiation and its function in Rictor-deficient osteoclasts were indistinguishable from those in control cells, suggesting that mTORC2 does not play a critical role in RANKL-dependent osteoclastogenesis in vitro. Although mTORC2 indirectly promotes osteoclastogenesis by modulating RANKL expression in osteoblasts, there is no evidence supporting the role of $\mathrm{mTORC} 2$ in bone resorption and bone homeostasis, through its expression in osteoclast lineage cells in vivo (Chen et al., 2015). An in vitro pharmacological study shows the possibility that mTORC2 regulates osteoclast fusion through Akt signaling (Tiedemann et al., 2017). However, due to the lack of an mTORC2-specific inhibitor and its widespread expression, it is noteworthy to demonstrate the functional relevance of mTORC2 on osteoclastogenesis, bone resorption, and bone remodeling in a cell-specific manner, by generating osteoclast lineage-specific Rictor-deficient mice.

It should be also noted that mTORC1 activity was markedly impaired during the course of osteoclast differentiation, in accordance with the marked upregulation of Hamartin (Tsc1) detected in this study. It has been shown that RANKL induces direct dephosphorylation of mTOR by calcineurin, leading to the downregulation of mTORC1 activity during osteoclastogenesis (Huynh and Wan, 2018). Our study demonstrated that augmentation of the protein level of a potent mTORC1 inhibitor, Hamartin (Tsc1), contributes to the downregulation of mTORC1 activity through RANKL stimulation during the course of osteoclast differentiation. However, further studies are needed to elucidate the exact mechanism(s) underlying RANKL-mediated upregulation of Hamartin (Tsc1) in osteoclasts.

In conclusion, the results of this study support the assertion that $\mathrm{mTORC} 1$, rather than mTORC2, is functionally expressed in osteoclast lineage cells to repress osteoclast differentiation during bone resorption and remodeling under pathological conditions. These data suggest that mTORC1 signal could be a future target for drug discovery and development for the treatment and therapy 

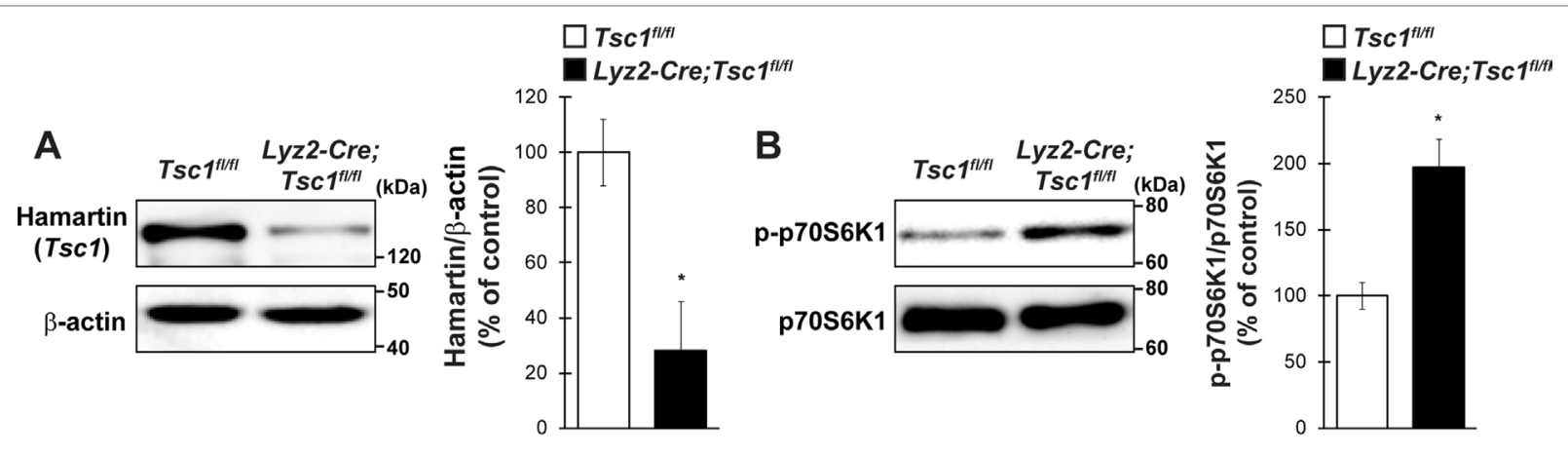

C

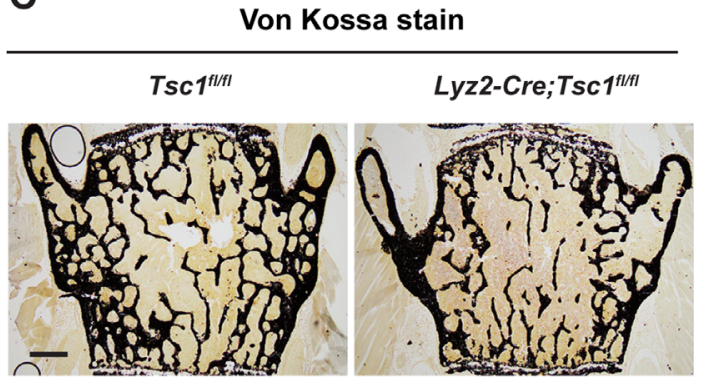

D

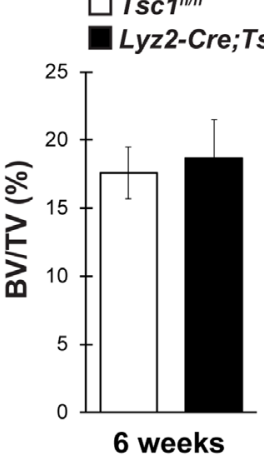

F

E
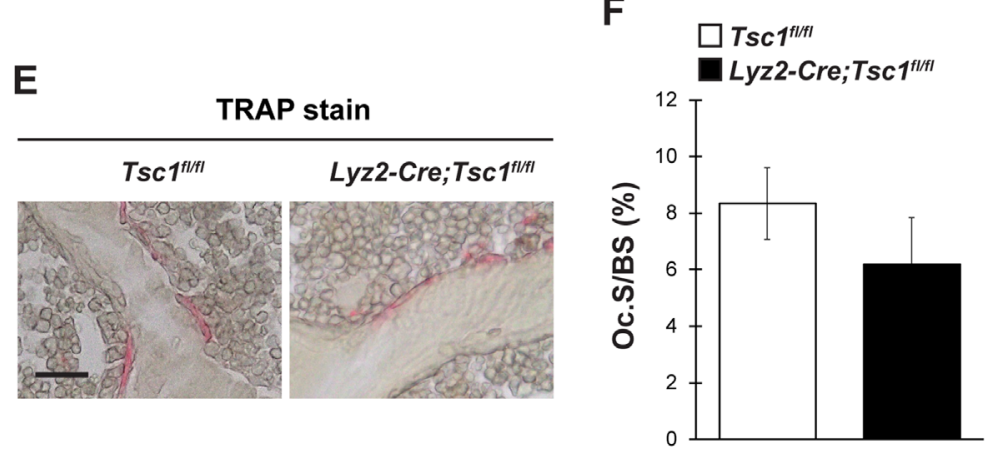

H

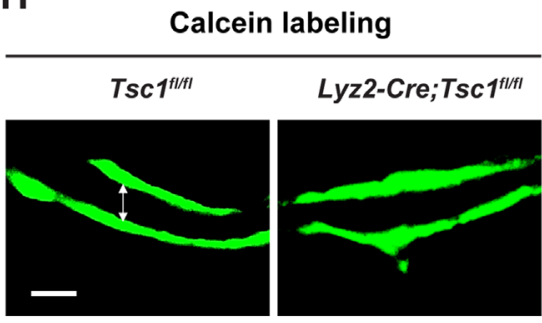

I

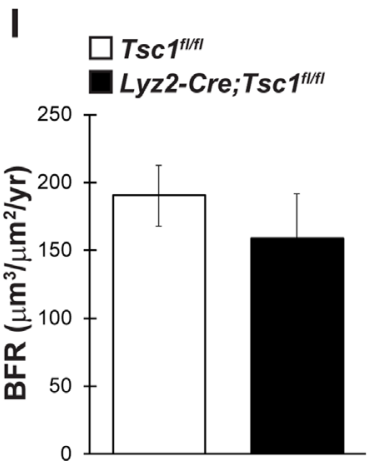

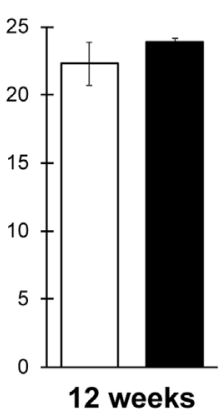

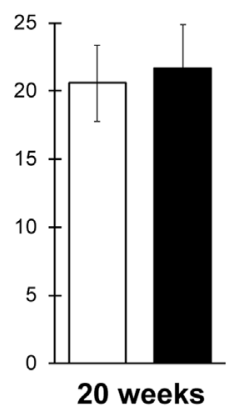

G

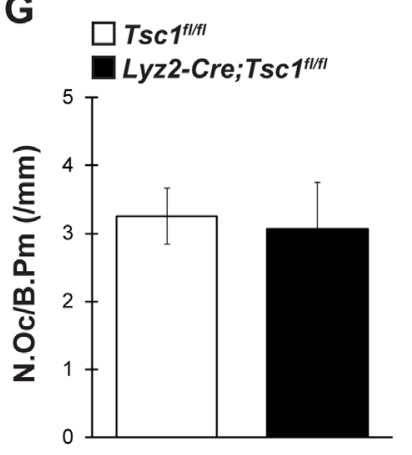

J

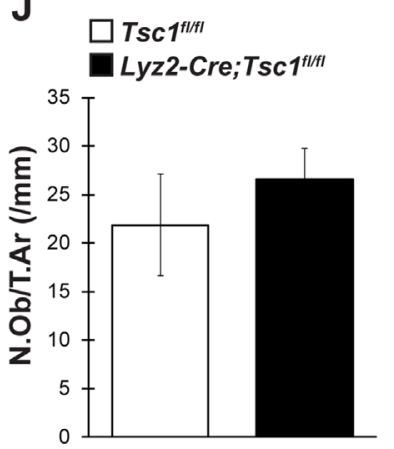

FIGURE 3 | TSC1 expressed by osteoclast lineage cells is dispensable for bone resorption and bone mass under physiological condition. (A) Hamartin (TSC1) expression in BMMs of control and Lyz2-Cre;Tsc1fl/fl mice $(n=3)$. (B) Phosphorylation level of p70S6K1 in BMMs of control and Lyz2-Cre; Tsc1fl/fl mice ( $n=3$ ). (C) von Kossa staining, (D) BV/TV (6-, 12-, and 20-week-old), (E) TRAP staining, (F) Oc.S/BS, (G) N.Oc/B.Pm, (H) calcein double labeling, (I) BFR, and (J) N.Ob/T. Ar of vertebrae of control and Lyz2-Cre; Tsc1fl/fl mice at 12-week-old (control, $n=5$; Lyz2-Cre;Tsc1fl/fl, $n=6)$. (C) Bar = 200 $\mu \mathrm{m}$, (E) bar = $50 \mu \mathrm{m}$, and (H) bar = 10 $\mu \mathrm{m}$. (H) Double-headed white arrow indicates the distance between calcein double labeling. All data were analyzed by the two-tailed Student $t$-test. 
A
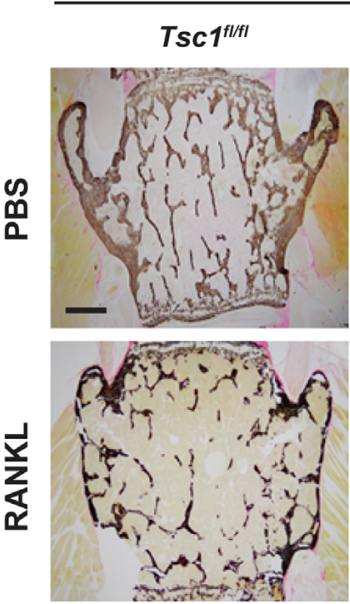

C

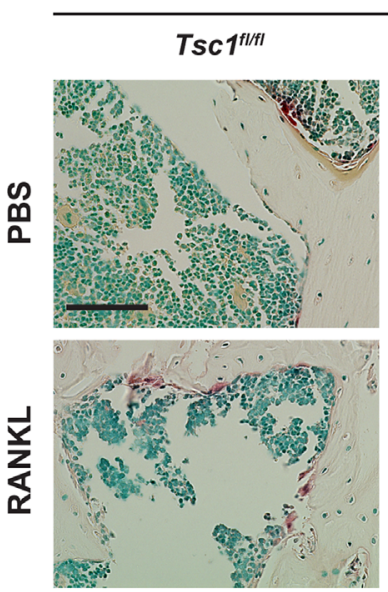

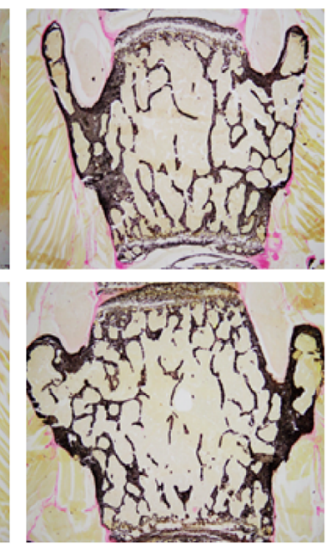

B

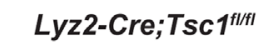

RAP stain

Lyz2-Cre;Tsc1 ${ }^{\text {fl/fl }}$
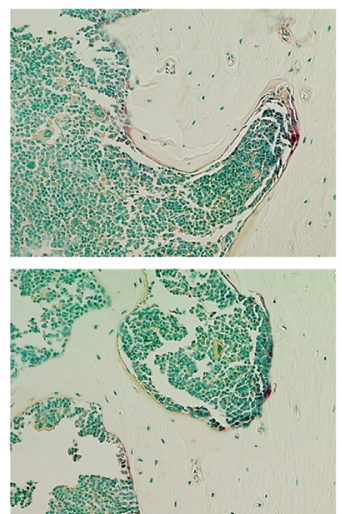

PBS

RANKL

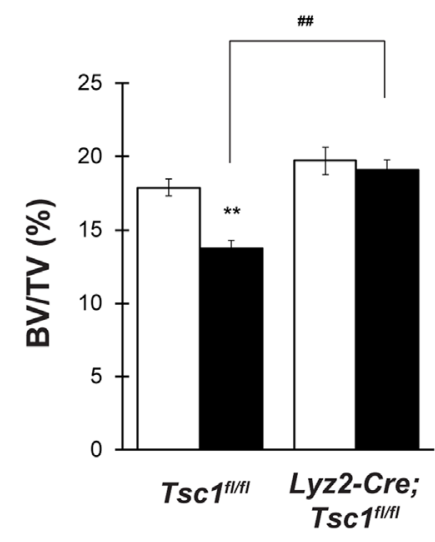

D

$\square$ PBS

RANKL

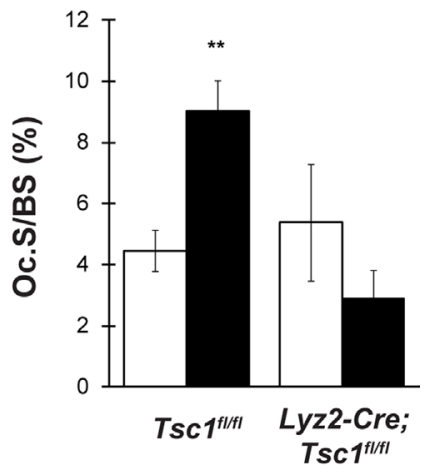

E $\square$ PBS

RANKL

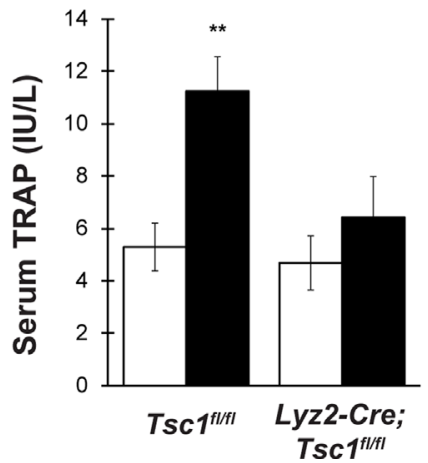

F $\square$ PBS

RANKL

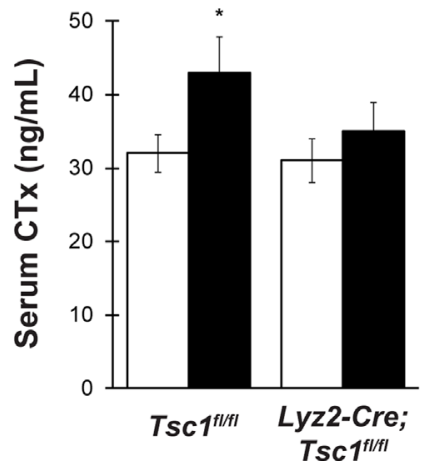

FIGURE 4 | mTORC1 in osteoclast lineage cells is implicated in RANKL-induced bone loss. Control mice and Lyz2-Cre; Tsc $1 f / f l$ mice were intraperitoneally administrated with GST-RANKL at $2 \mathrm{mg} / \mathrm{kg}$ daily for 2 days, and subsequently, mice were sacrificed $12 \mathrm{~h}$ after the final injection, followed by determination of bone phenotypes (control-PBS, $n=6$; control-RANKL, $n=6$; Lyz2-Cre; Tsc1fl/fl-PBS, $n=7$; Lyz2-Cre;Tsc1fl/fl-RANKL, $n=8$ ). (A) von Kossa staining, (B) BV/TV, (C) TRAP staining, and (D) Oc.S/BS of the vertebrae of PBS- or RANKL-injected mice. Serum (E) TRAP and (F) CTx levels of PBS- or RANKL-injected mice. All data were analyzed by the two-way ANOVA with Bonferroni/Dunnett post hoc test. ${ }^{\star} P<0.05,{ }^{* *} P<0.01$, significantly different from the value obtained for PBS-injected mice. ${ }^{\# P} P<0.01$ significantly different from the value obtained for RANKL-injected control mice. (A) Bar $=200 \mu \mathrm{m}$ and (C) bar $=50 \mu \mathrm{m}$. 
of a variety of metabolic bone diseases caused by abnormal activation of osteoclasts, such as osteoporosis, lytic bone metastasis, and rheumatoid arthritis. Moreover, considering that the mTORC1 pathway could be a key modulator of inflammatory conditions such as aging and rheumatic disease (Tomimori et al., 2009; Johnson et al., 2013), our findings may contribute to opportunities for the development of drugs targeting age-related pathologies.

\section{ETHICS STATEMENT}

The protocol used here meets the guideline of the Japanese Society for Pharmacology and was approved by the Committee for Ethical Use of Experimental Animals at Kanazawa University.

\section{AUTHOR CONTRIBUTIONS}

Study design: EH. Study conduct: MH, KO, TY, TI, GP, KF, TH, HK, KT, and MM. Data collection: MH, KO, TY, TI, GP, KF, TH, and HK. Data analysis: MH, TY, TI, KO, GP, KF, TH, and HK. Data interpretation: MH, KO, TY, TI, GP, KF, TH, HK, KK and EH. Drafting manuscript: EH. Approving the final version of the manuscript: All authors.

\section{REFERENCES}

Brown, E. J., Albers, M. W., Shin, T. B., Ichikawa, K., Keith, C. T., Lane, W. S., et al. (1994). A mammalian protein targeted by G1-arresting rapamycin-receptor complex. Nature 369 (6483), 756-758. doi: 10.1038/369756a0

Chen, J., Holguin, N., Shi, Y., Silva, M. J., and Long, F. (2015). mTORC2 signaling promotes skeletal growth and bone formation in mice. J. Bone Miner. Res. 30 (2), 369-378. doi: 10.1002/jbmr.2348

Chen, J., and Long, F. (2014). mTORC1 signaling controls mammalian skeletal growth through stimulation of protein synthesis. Development 141 (14), 28482854. doi: $10.1242 /$ dev. 108811

Chen, J., and Long, F. (2018). mTOR signaling in skeletal development and disease. Bone Res. 6, 1. doi: 10.1038/s41413-017-0004-5

Dai, Q., Xie, F., Han, Y., Ma, X., Zhou, S., Jiang, L., et al. (2017). Inactivation of regulatory-associated protein of $\mathrm{mTOR}$ (raptor)/mammalian target of rapamycin complex 1 (mTORC1) signaling in osteoclasts increases bone mass by inhibiting osteoclast differentiation in mice. J. Biol. Chem. 292 (1), 196-204. doi: $10.1074 /$ jbc.M116.764761

Feng, X., and McDonald, J. M. (2011). Disorders of bone remodeling. Annu. Rev. Pathol. 6, 121-145. doi: 10.1146/annurev-pathol-011110-130203

Fukasawa, K., Park, G., Iezaki, T., Horie, T., Kanayama, T., Ozaki, K., et al. (2016). ATF3 controls proliferation of osteoclast precursor and bone remodeling. Sci. Rep. 6, 30918. doi: 10.1038/srep30918

Guertin, D. A., Stevens, D. M., Thoreen, C. C., Burds, A. A., Kalaany, N. Y., Moffat, J., et al. (2006). Ablation in mice of the mTORC components raptor, rictor, or mLST8 reveals that mTORC2 is required for signaling to Akt-FOXO and PKCalpha, but not S6K1. Dev. Cell. 11 (6), 859-871. doi: 10.1016/j. devcel.2006.10.007

Harada, S., and Rodan, G. A. (2003). Control of osteoblast function and regulation of bone mass. Nature 423 (6937), 349-355. doi: 10.1038/nature01660

Hinoi, E., Takarada, T., Uno, K., Inoue, M., Murafuji, Y., and Yoneda, Y. (2007). Glutamate suppresses osteoclastogenesis through the cystine/glutamate antiporter. Am. J. Pathol. 170 (4), 1277-1290. doi: 10.2353/ajpath.2007.061039

Huynh, H., and Wan, Y. (2018). mTORC1 impedes osteoclast differentiation via calcineurin and NFATc1. Comm. Biol. 1 (1), 29. doi: 10.1038/ s42003-018-0028-4

\section{FUNDING}

This work was supported in part by the Japan Society for the Promotion of Science (16H05131, 17KT0051, and 18H04971 to $\mathrm{EH}$ ) and the Japan Agency for Medical Research and Development (17824969 to EH).

\section{ACKNOWLEDGMENTS}

We highly thank Drs. S.L. Teitelbaum (Washington University, St. Louis, MO, USA) for kindly providing GST-RANKL construct and T. Kitamura (Tokyo University, Tokyo, Japan) for generously providing PLAT-E cells.

\section{SUPPLEMENTARY MATERIAL}

The Supplementary Material for this article can be found online at: https://www.frontiersin.org/articles/10.3389/fphar.2019.00684/ full\#supplementary-material

SUPPLEMENTAL FIGURE 1 | Actin ring formation assay. BMMs from Tsc1fl/fl mice were retrovirally infected with Cre recombinase, and subsequent stimulation with RANKL, followed by actin ring formation assay $(n=3)$. ${ }^{*} P<0.05$, significantly different from the value obtained in control cells.

Iezaki, T., Horie, T., Fukasawa, K., Kitabatake, M., Nakamura, Y., Park, G., et al. (2018). Translational control of Sox9 RNA by mTORC1 Contributes to skeletogenesis. Stem Cell. Rep. 11 (1), 228-241. doi: 10.1016/j.stemcr.2018.05.020

Iezaki, T., Onishi, Y., Ozaki, K., Fukasawa, K., Takahata, Y., Nakamura, Y., et al. (2016). The Transcriptional modulator interferon-related developmental regulator 1 in osteoblasts suppresses bone formation and promotes bone resorption. J. Bone Miner. Res. 31 (3), 573-584. doi: 10.1002/jbmr.2720

Jacinto, E., Loewith, R., Schmidt, A., Lin, S., Ruegg, M. A., Hall, A., et al. (2004). Mammalian TOR complex 2 controls the actin cytoskeleton and is rapamycin insensitive. Nat. Cell. Biol. 6 (11), 1122-1128. doi: 10.1038/ncb1183

Johnson, S. C., Rabinovitch, P. S., and Kaeberlein, M. (2013). mTOR is a key modulator of ageing and age-related disease. Nature 493 (7432), 338-345. doi: 10.1038 /nature11861

Karsenty, G., Kronenberg, H. M., and Settembre, C. (2009). Genetic control of bone formation. Annu. Rev. Cell. Dev. Biol. 25, 629-648. doi: 10.1146/annurev. cellbio.042308.113308

Long, X., Lin, Y., Ortiz-Vega, S., Yonezawa, K., and Avruch, J. (2005). Rheb binds and regulates the mTOR kinase. Curr. Biol. 15 (8), 702-713. doi: 10.1016/j. cub.2005.02.053

Murakami, M., Ichisaka, T., Maeda, M., Oshiro, N., Hara, K., Edenhofer, F., et al. (2004). mTOR is essential for growth and proliferation in early mouse embryos and embryonic stem cells. Mol. Cell Biol. 24 (15), 6710-6718. doi: 10.1128/ MCB.24.15.6710-6718.2004

Nakamura, Y., Hinoi, E., Iezaki, T., Takada, S., Hashizume, S., Takahata, Y., et al. (2013). Repression of adipogenesis through promotion of Wnt/beta-catenin signaling by TIS7 up-regulated in adipocytes under hypoxia. Biochim. Biophys. Acta 1832 (8), 1117-1128. doi: 10.1016/j.bbadis.2013.03.010

Nojima, H., Tokunaga, C., Eguchi, S., Oshiro, N., Hidayat, S., Yoshino, K., et al. (2003). The mammalian target of rapamycin (mTOR) partner, raptor, binds the mTOR substrates p70 S6 kinase and 4E-BP1 through their TOR signaling (TOS) motif. J. Biol. Chem. 278 (18), 15461-15464. doi: 10.1074/jbc.C200665200

Sabatini, D. M. (2017). Twenty-five years of mTOR: uncovering the link from nutrients to growth. Proc. Natl. Acad. Sci. U. S. A 114 (45), 11818-11825. doi: 10.1073/pnas.1716173114

Sabatini, D. M., Erdjument-Bromage, H., Lui, M., Tempst, P., and Snyder S. H. (1994). RAFT1: a mammalian protein that binds to FKBP12 in a 
rapamycin-dependent fashion and is homologous to yeast TORs. Cell 78 (1), 35-43. doi: 10.1016/0092-8674(94)90570-3

Shiota, C., Woo, J. T., Lindner, J., Shelton, K. D., and Magnuson, M. A. (2006). Multiallelic disruption of the rictor gene in mice reveals that mTOR complex 2 is essential for fetal growth and viability. Dev. Cell. 11 (4), 583-589. doi: 10.1016/j.devcel.2006.08.013

Teitelbaum, S. L., and Ross, F. P. (2003). Genetic regulation of osteoclast development and function. Nat. Rev. Genet. 4 (8), 638-649. doi: 10.1038/ nrg 1122

Tiedemann, K., Le Nihouannen, D., Fong, J. E., Hussein, O., Barralet, J. E., and Komarova, S. V. (2017). Regulation of osteoclast growth and fusion by mTOR/ raptor and mTOR/rictor/Akt. Front. Cell. Dev. Biol. 5, 54. doi: 10.3389/ fcell.2017.00054

Tomimori, Y., Mori, K., Koide, M., Nakamichi, Y., Ninomiya, T., Udagawa, N., et al. (2009). Evaluation of pharmaceuticals with a novel 50-hour animal model of bone loss. J. Bone Miner. Res. 24 (7), 1194-1205. doi: 10.1359/jbmr.090217

Wang, L., Hinoi, E., Takemori, A., Nakamichi, N., and Yoneda, Y. (2006). Glutamate inhibits chondral mineralization through apoptotic cell death mediated by retrograde operation of the cystine/glutamate antiporter. J. Biol. Chem. 281 (34), 24553-24565. doi: 10.1074/jbc.M600939200

Yamamoto, T., Hinoi, E., Fujita, H., Iezaki, T., Takahata, Y., Takamori, M., et al. (2012). The natural polyamines spermidine and spermine prevent bone loss through preferential disruption of osteoclastic activation in ovariectomized mice. $\mathrm{Br}$ J. Pharmacol. 166 (3), 1084-1096. doi: 10.1111/j.1476-5381.2012.01856.x

Yan, B., Zhang, Z., Jin, D., Cai, C., Jia, C., Liu, W., et al. (2016). mTORC1 regulates PTHrP to coordinate chondrocyte growth, proliferation and differentiation. Nat. Commun. 7, 11151. doi: 10.1038/ncomms11151

Zhang, Y., Xu, S., Li, K., Tan, K., Liang, K., Wang, J., et al. (2017). mTORC1 inhibits NF-kappaB/NFATc1 signaling and prevents osteoclast precursor differentiation, in vitro and in mice. J. Bone Miner. Res. 32 (9), 1829-1840. doi: 10.1002/jbmr.3172

Conflict of Interest Statement: The authors declare that the research was conducted in the absence of any commercial or financial relationships that could be construed as a potential conflict of interest.

Copyright (c) 2019 Hiraiwa, Ozaki, Yamada, Iezaki, Park, Fukasawa, Horie, Kamada, Tokumura, Motono, Kaneda and Hinoi. This is an open-access article distributed under the terms of the Creative Commons Attribution License (CC $B Y)$. The use, distribution or reproduction in other forums is permitted, provided the original author(s) and the copyright owner(s) are credited and that the original publication in this journal is cited, in accordance with accepted academic practice. No use, distribution or reproduction is permitted which does not comply with these terms. 